\title{
O perfil epidemiológico de gestantes atendidas nas unidades básicas de saúde de Gurupi, Tocantins*
}

\section{Epidemiological profile of pregnant women answered in basic unit Gurupí health, Tocantins}

Marcos Gontijo da Silva' Érica Eugênio Lourenço Gontijo'

Dayane da Silva Ferreira² Fernanda Soares Carvalho ${ }^{2}$ Ana Maria de Castro ${ }^{3}$
Recebido em: 15/04/2015.

Aprovado em: 13/09/2015.

1 Professores Adjuntos do curso de medicina do Centro Universitário UNIRG, Brasil, Palmas. Telefone: (63) 8126-9197. E-mail: gontijobio@ yahoo.com.br; ericagontijo1@yahoo.com.br

Bacharéis em enfermagem pelo Centro Universitário UNIRG, Brasil, Gurupi. Telefones: (63) 84161938, (63) 9211-6885. E-mail: melgomes_f@hotmail.com; nandahta2006@hotmail.com.

3 Professora Associada da Universidade Federal de Goiás. Programa de Pós-Graduação em Medicina Tropical no IPTSP. Laboratório da Relação Parasito Hospedeiro LAERPH, IPTSP-UFG, Brasil, Goiânia. E-mail: amaria.ana@gmail.com.

\section{Resumo}

Este artigo propôs-se a traçar o perfil clínico e sociodemográfico das gestantes atendidas pelo SUS (Sistema Único de Saúde) no Município de Gurupi, Tocantins. Foi feita uma análise retrospectiva nas UBS (Unidades Básicas de Saúde) da cidade. Foram avaliadas 6583 gestantes e selecionadas 1049 mulheres. Foram coletados dados demográficos, sociais e clínicos. Os dados foram tabulados no programa Epi-Info 3.3.2 sendo aplicada estatística descritiva com IC95\%. Foi observado que 47,0\% não se submeteram a seis consultas pré-natais, $83,1 \%$ eram negras ou pardas e $46,4 \%$ não tinham profissão. Foi encontrada toxoplasmose aguda em 5,0\% (52/1049), sífilis em 3,6\% (38/1049), hepatite B em 2,7\% (28/1049), herpes simplex vírus em 2,0\% (21/1049), HIV em 1,9\% (20/1049), hipertensão em 15,6\% (164/1049), doença neurológica em 1,2\% $(13 / 1049)$ e diabetes em $1,1 \%$ (12/1049), cefaléia 38\%, dor no baixo ventre $35,5 \%$ e leucorreia 19,2\%. Concluímos que a prevalência de doenças infecciosas e não infecciosas nas gestantes foi alta.

Palavras-chave: Pré-Natal. Transmissão vertical. Intercorrências gestacionais. Gravidez. Perfil sociodemográfico.

\begin{abstract}
This set out to trace the clinical and socio-demographic profile of pregnant women served by the SUS (Brazilian Health System) in the municipality of Gurupi, Tocantins. A retrospective analysis was done in UBS (Basic Health Units) of the city. 6583 pregnant women were evaluated and selected 1049 women. Demographic, social and clinical data were collected. Data were tabulated on Epi-Info 3.3.2 program being applied descriptive statistics with 95\%. It was observed that $47.0 \%$ did not undergo the six prenatal visits, $83.1 \%$ were black or brown and $46.4 \%$ had no profession. It was found acute toxoplasmosis $5.0 \%$ (52/1049), syphilis 3.6\% (38/1049), hepatitis B 2.7\% (28/1049), herpes simplex virus $2.0 \%$ (21/1049), HIV 1.9\% (20/1049), hypertension in 15.6\% (164/1049), neurological disease $1.2 \%$ (13/1049), diabetes $1.1 \%(12 / 1049)$. headache $38 \%$, pain in the lower abdomen $35.5 \%$ and $19.2 \%$ vaginal discharge. We conclude that the prevalence of infectious and non-infectious diseases in pregnant women in the Gurupi municipality is extremely high.
\end{abstract}

Keywords: Prenatal. Vertical transmission. Pregnancy complications. Pregnancy. Sociodemographic profile. 


\section{Introdução}

Durante a gestação, o equilíbrio fisiológico do organismo materno é bastante alterada, tornando-se, geralmente, um dos poucos momentos de contato com os serviços de saúde e uma boa oportunidade para o rastreamento de enfermidades (COSTA et al., 2010).

Nesse período inúmeras mudanças começam a ocorrer no corpo feminino que sofre diversas alterações endócrinas e imunológicas. O sistema imune materno está em íntimo contato com o feto, que pode ser comparado a um aloenxerto, pois possui 50\% de material genético paterno (VIANNA, 2009). O embrião representa transplante para o sistema imune materno estando, portanto, vulnerável à rejeição ou à tolerância imunológica. O sistema imunológico materno para se manter tolerante aos antígenos sofre algumas alterações. Os hormônios que são produzidos na gestação, como os estrogênios, em altas concentrações, contribuirão para a diminuição de autoanticorpos, o que torna a mulher mais vulnerável a diversas doenças (BARROS, 2006; NEVES et al., 2007). Os esteroides sexuais têm efeito inibitório na atividade das células B (NEVES et al., 2007).

Várias outras alterações ocorrem para manter o sistema imunológico materno tolerante aos antígenos paternos de histocompatibilidade expressos pelo feto com a estimulação do sistema imunológico inato e supressão do sistema imunológico adaptativo (VIANNA, 2009). Ocorre o aumento da percentagem dos granulócitos e diminuição dos linfócitos (LUPPI et al., 2002). Os linfócitos T apresentam-se inativados com diminuição da produção de IL-6 (LUPPI et al., 2002). O HLA-G, membro do MHC da classe I, expresso nas células trofoblásticas, inibe a função das células NK e a maturação das células dendríticas, desempenhando função importante na tolerância fetal às células maternas (BARROS, 2006).

As modificações do sistema imunológico durante a gravidez aumentam a probabilidade de ocorrência de complicações. A gestação está entre as situações mais peculiares do ponto de vista imunológico. Modificações na produção de substâncias próprias desse sistema atuam de forma direta ou indireta, podendo expor a gestante e o embrião/feto a infecções que podem provocar graves lesões ou mesmo aborto (PROENÇA, 2011).

As doenças infecciosas associadas à gravidez alteram a saúde da mulher, podem interferir na função reprodutora desta e geram influência negativa sobre o feto
(PORTUGAL, 2000). As infecções congênitas estão entre as principais causas de morbidade e mortalidade no período neonatal (PADMAVATHY et al., 2013). Essas infecções implicam resultado fetal desfavorável podendo desencadear abortos, retardo do crescimento fetal, prematuridade e/ou anomalias congênitas (DE-PASCHALE et al., 2014). O conhecimento da prevalência e etiologia destas são relevantes, pois podem desencadear prejuízos para o feto e/ou recém-nascido.

A gravidez promove desequilíbrio metabólico, circulatório, neurológico, renal que predispõe a mulher a desenvolver quadro clínico no limiar patológico e que pode causar complicações maternas ou fetais. A principal complicação não infecciosa que mais causa morte materna no Brasil consiste na hipertensão arterial, podendo também desencadear danos ao feto como, baixo peso ao nascer, risco de desenvolver doenças pulmonares, e suprimento inadequado de oxigênio (WHO, 2010). A gravidez altera hábitos alimentares e a sensibilidade à insulina e produção de hormônios. Essas mudanças alteram o metabolismo de carboidrato e as necessidades de insulina, podendo desencadear diabetes mellitus gestacional (DMG). Os bebês de gestantes diabéticas são considerados grandes e pesados para a idade gestacional e podem desenvolver malformações congênitas em 5 a 10\% dos casos (ZIEGEL; CRANLEY, 1985).

Devido à turbulência desse período se torna de fundamental importância o acompanhamento médico materno durante esse período, de forma a evitar ou minimizar as intercorrências comuns dessa fase. Por isso o pré-natal é de fundamental importância para o acompanhamento da gestação, evitando condições de risco tanto para a mãe quanto para seu filho e deve ser direcionado para suprir as necessidades das gestantes por meio de consultas mensais e oferecer recursos e procedimentos adequados para o sucesso da gestação (COSTA et al., 2010).

Dentre os programas formulados pelo Ministério da Saúde (MS), destaca-se o Programa de Assistência Integral a Saúde da Mulher (PAISM), criado na década de 1980, com a finalidade de prestar assistência à mulher em todas as etapas do seu ciclo vital, por meio de atividades clínico-ginecológicas, tais como: identificação, diagnóstico e tratamento das doenças sistêmicas e do aparelho reprodutivo; assistência pré-natal, ao parto e puerpério; além de atividades educativas que proporcionem às mulheres maior conhecimento sobre o próprio corpo, inclu- 
sive para que elas possam vivenciar melhor a sexualidade, influenciando diretamente na qualidade de vida delas (COSTA et al., 2010).

O Brasil é um país de nível continental e muito heterogêneo nas mais diversas áreas, incluindo a assistência pré-natal. Cada região tem suas peculiaridades e necessidades, bem como suas doenças mais prevalentes, $\mathrm{o}$ que obriga atendimento diferenciado para atender as necessidades regionais. Por isso, é de suma importância ter conhecimento do perfil clínico e sociodemográfico e clínico de gestantes de uma cidade no intuito de otimizar o atendimento de acordo com cada necessidade específica.

Gurupi é a terceira maior cidade do estado do Tocantins, possuindo 81.792 habitantes, localizada no sul do estado (INSTITUTO BRASILEIRO DE GEOGRAFIA E ESTATÍSTICA, 2013). O município possui taxa de fecundidade de 2,0 filhos por mulher, e uma taxa de mortalidade até um ano de vida de 12,7 a cada 1000 nascidos vivos (INSTITUTO DE PESQUISA ECONÔMICA APLICADA, 2013).

Devido a isso se acredita que a realidade das gestantes do Município de Gurupi, Tocantins, seja provavelmente diferente de outras regiões. Assim observa-se a grande importância de conhecer as características locais para a implantação de medidas preventivas direcionadas e mais eficazes. Por isso esse trabalho se propõe a avaliar o perfil epidemiológico de gestantes atendidas nas unidades básicas de saúde de Gurupi, Tocantins.

\section{Metodologia}

O estudo consistiu em tipo exploratório descritivo de abordagem quantitativa, com emprego da técnica de observação indireta por meio da análise retrospectiva das fichas de atendimento do Serviço de Obstetrícia conduzido na cidade de Gurupi, localizadas no estado do Tocantins, Brasil. A coleta de dados foi feita em 11 UBS da zona urbana da cidade.

As gestantes atendidas nas UBSs do município de Gurupi são triadas sorológicamente para diversas infecções como HIV, sífilis, toxoplasmose e exporadicamente quando existe suspeita para rubéola, Citomegalovírus e gonorreia. Os exames são realizados em laboratórios credenciados à secretaria municipal de saúde no município.

As informações foram obtidas acessando banco de dados das UBSs do município nos últimos cinco anos. Foram selecionadas mulheres que realizaram o pré-natal a partir do ano de 2008, sendo investigadas todas as gestantes das UBSs, porém só foram incluídas na pesquisa as gestantes que tinham seus dados completos e que fossem moradoras do município. Foram excluídas todas as gestantes de outros municípios e com dados incompletos.

A coleta de dados se deu após a aprovação pelo Comitê de Ética em Pesquisa do Centro Universitário UNIRG com número 394846 de acordo com a Resolução 196 do Conselho Nacional de Saúde (2006) e a aprovação da secretária de saúde do município e da direção de cada UBS. Foi mantido total sigilo quanto à identidade das participantes.

Foram avaliadas 6.583 gestantes, porém excluídos todos os formulários que não tinham resultados da sorologia para toxoplasmose, sendo selecionadas para a pesquisa 1.049 mulheres que constituíram o grupo amostral do inquérito e que realizaram sorologia para toxoplasmose na gestação. Foram coletados dados demográficos (faixa etária, etnia), sociais (grau de instrução, estado civil, ocupação, moradia, número de gestações, renda familiar), clínicos (presença de infecções como: HIV, toxoplasmose, Sífilis, citomegalovírus, rubéola, gonorreia e infecção urinária), tipo de parto (cesáreo ou normal), número de consultas pré-natal $(<6$ ou $\geq 6)$ e principais intercorrências na gestação.

Os dados foram tabulados no programa Epi-Info 3.3.2 sendo aplicada estatística descritiva, com emprego das frequências absoluta e percentual, evidenciados em tabelas. Os dados foram discutidos com base na síntese de indicadores sociais, de documentos da situação de saúde no Brasil e demais referências que analisam o tema em questão.

\section{Resultados}

Foram pesquisadas 1.049 gestantes atendidas entre o período de janeiro de 2008 a dezembro de 2013 e excluídos 5.534 que não atenderam os critérios de inclusão. Quanto a informações coletadas, essas foram agrupadas em: caracterização sociodemográfica e de procedimento; caracterização das doenças infecciosas; caracterização das doenças não infecciosas e caracterização das principais queixas e intercorrências.

Quanto às características sociodemográficas e de procedimento, destacou-se o fato de quase a metade das gestantes não se submeteram às seis consultas pré-natais recomendadas pela OMS, a maioria eram negras ou pardas e quase a metade não tinha profissão (Tabela 1). 
Tabela 1 - Caracterização sociodemográfica e de procedimento nas gestantes pesquisadas em Gurupi, Tocantins, Brasil, 2014.

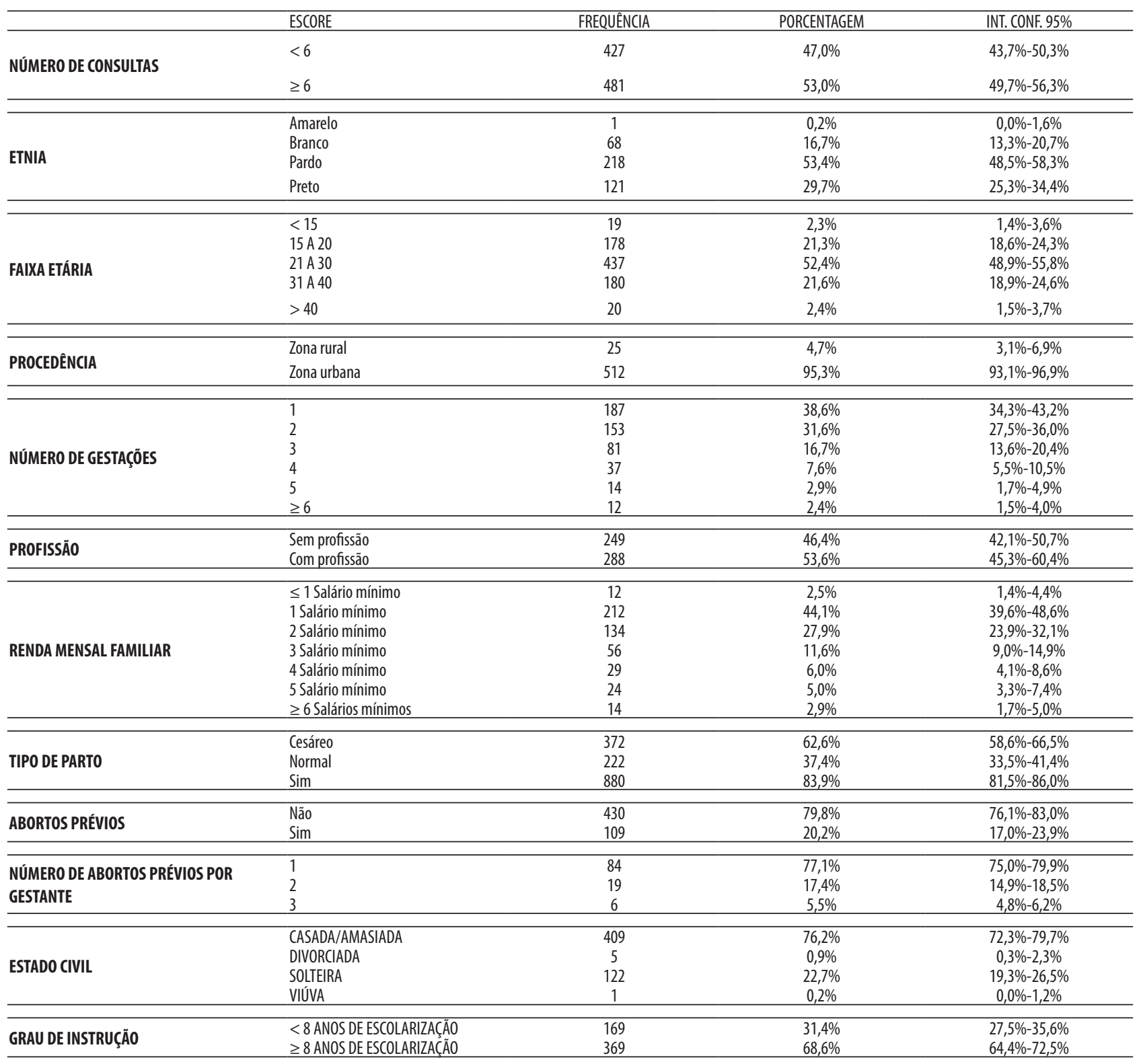

Quanto à prevalência de doenças infecciosas que acometiam as gestantes pesquisadas, foi observada a presença das seguintes infecções: Toxoplasmose, sífilis, Citomegalovirose, Rubéola, AIDS/SIDA (Síndrome da Imunodeficiência Adquirida), Gonorreia, Hepatites B e C e Infecção urinária (Tabela 2).

Tabela 2 - Caracterização das infecções gestacionais nas gestantes pesquisadas em Gurupi, Tocantins, Brasil, 2014.

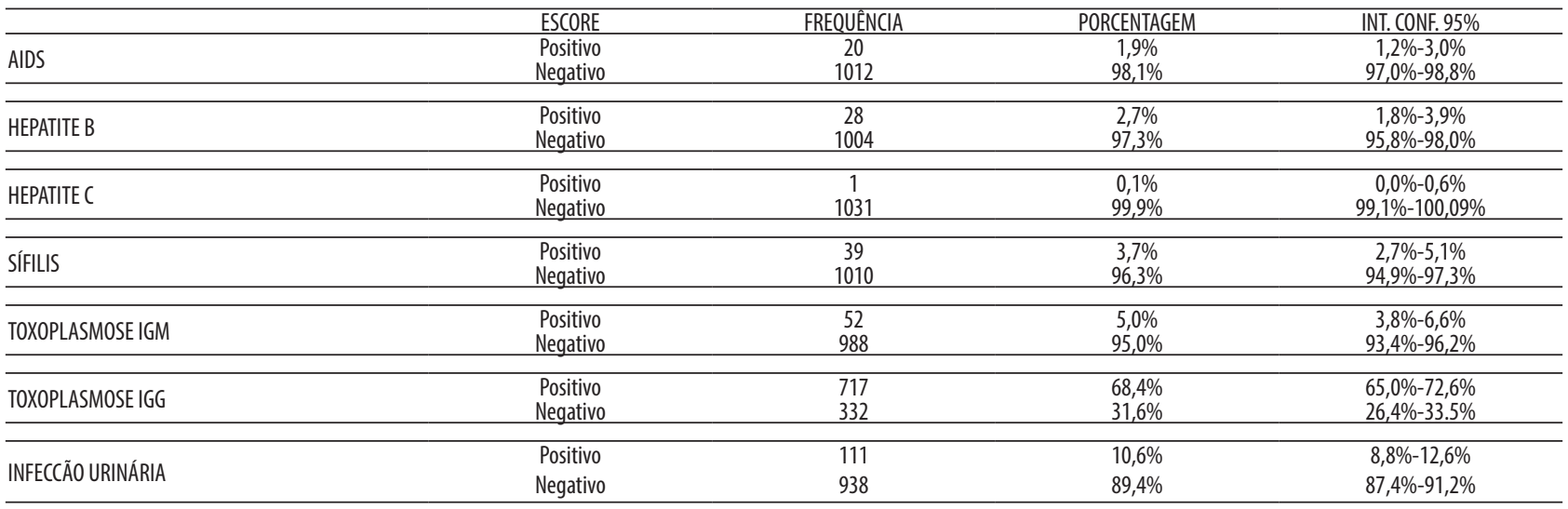


Quanto à prevalência de doenças não infecciosas que acometiam as gestantes pesquisadas, foi observado a presença das seguintes alterações: Hipertensão, diabetes, doença cardíaca, renal e neurológica (Tabela 3).

Tabela 3 - Caracterização das doenças não infecciosas encontradas nas gestantes pesquisadas em Gurupi, Tocantins, Brasil, 2014.

\begin{tabular}{|c|c|c|c|c|c|}
\hline Categoria & $\begin{array}{c}\text { Frequência } \\
\text { absoluta }\end{array}$ & $\%$ & Classificação & $\begin{array}{l}\text { Frequência } \\
\text { relativa }\end{array}$ & $\%$ \\
\hline $\begin{array}{l}\text { Problema } \\
\text { circulatório }\end{array}$ & 164 & $15,6 \%$ & Hipertensão & 164 & $100 \%$ \\
\hline $\begin{array}{l}\text { Distúrbio } \\
\text { metabólico }\end{array}$ & 12 & $1,1 \%$ & Diabetes & 12 & $100 \%$ \\
\hline \multirow{3}{*}{ Cardiopatias } & \multirow{3}{*}{10} & \multirow{3}{*}{$1,0 \%$} & Arritmia & 4 & $40,0 \%$ \\
\hline & & & Miocardite & 4 & $40,0 \%$ \\
\hline & & & Sopro sistólico & 2 & $20,0 \%$ \\
\hline \multirow{3}{*}{ Neuropatias } & \multirow{3}{*}{13} & \multirow{3}{*}{$1,2 \%$} & Epilepsia & 6 & $46,15 \%$ \\
\hline & & & Retardo mental & 4 & $30,77 \%$ \\
\hline & & & Transtorno bipolar & 3 & $23,07 \%$ \\
\hline \multirow{4}{*}{ Doença renal } & \multirow{4}{*}{6} & \multirow{4}{*}{$0,6 \%$} & Cólica renal & 1 & $16,7 \%$ \\
\hline & & & Incontinência Urinária & 3 & $50,0 \%$ \\
\hline & & & Infecção renal & 1 & $16,7 \%$ \\
\hline & & & Litíase & 1 & $16,7 \%$ \\
\hline
\end{tabular}

Quanto às principais intercorrências que acometeram as gestantes pesquisadas, foi observado a presença das seguintes alterações: Cefaleia, dor no baixo-ventre, leucorreia e lombalgia (Tabela 4).

Tabela 4 - Caracterização das principais intercorrências encontradas nas gestantes pesquisadas em Gurupi, Tocantins, Brasil, 2014.

\begin{tabular}{lccc}
\hline INTERCORRÊNCIA & $\begin{array}{c}\text { FREQUÊNCIA } \\
\text { ABSOLUTA }\end{array}$ & PORCENTAGEM & INT. CONF. 95\% \\
\hline CEFALEIA & 399 & $38,0 \%$ & $35,1 \%-41,1 \%$ \\
DOR NO Baixo-ventre & 372 & $35,5 \%$ & $32,6 \%-38,5 \%$ \\
LEUCORREIA & 201 & $19,2 \%$ & $16,8 \%-21,7 \%$ \\
LOMBALGIA & 177 & $16,9 \%$ & $14,7 \%-19,3 \%$ \\
EDEMA NOS MEMBROS INFERIORES & 143 & $13,6 \%$ & $11,6 \%-15,9 \%$ \\
NÁUSEA & 129 & $12,3 \%$ & $10,4 \%-14,5 \%$ \\
SANGRAMENTO VAGINAL & 94 & $9,0 \%$ & $7,3 \%-10,9 \%$ \\
VERTIGEM & 80 & $7,6 \%$ & $6,1 \%-9,4 \%$ \\
EPIGASTRALGIA & 80 & $7,6 \%$ & $6,1 \%-9,4 \%$ \\
PIROSE & 77 & $7,3 \%$ & $5,9 \%-9,1 \%$ \\
INAPETÊNCIA/ASTENIA & 61 & $5,8 \%$ & $4,5 \%-7,5 \%$ \\
DISPNEIA & 45 & $4,3 \%$ & $3,2 \%-5,7 \%$ \\
INSÔNIA & 45 & $4,3 \%$ & $3,2 \%-5,7 \%$ \\
\hline
\end{tabular}

\section{Discussão}

O acesso à assistência pré-natal é considerado condição sine qua non para que a gestação transcorra sem problemas tanto para a mãe quanto para o filho (BRASIL,
2012). A assistência pré-natal deve ter início no primeiro trimestre de gravidez, com consultas médicas mensais para oferecer cobertura efetiva e ampla, de modo organizado e planejado, de forma a permitir o acompanhamento profilático efetivo (INSTITUTO BRASILEIRO DE GEOGRAFIA E ESTATÍSTIA, 2009).

O Ministério da Saúde preconiza a realização de consulta no primeiro trimestre, duas no segundo e três no terceiro (INSTITUTO BRASILEIRO DE GEOGRAFIA E ESTATÍSTIA, 2009; ANDREUCCI; CECATI, 2011). Identificou-se que $53 \%$ das pesquisadas realizaram seis ou mais consultas no pré-natal, e o restante das gestantes, quase metade (47\%) se submeteram a um número de consultas inferior ao recomendado pelo ministério da saúde. Ao comparar com os dados do estado do Tocantins, foi observado que apenas $39,1 \%$ se submetem a mais de 6 consultas pré-natais (BRASIL, 2009, COUTINHO et al., 2010; DOMINGUES et al., 2012). Em São Paulo, a realidade é diferente e $72,5 \%$ das gestantes são submetidas a 6 ou mais consultas no pré-natal (CUNHA, 2008). Segundo dados do IBGE, evidenciou-se aumento da proporção de nascidos vivos cujas mães realizaram seis ou mais consultas pré-natal, passando de 43,7\%, em 2000, para 54,5\%, em 2006 (INSTITUTO BRASILEIRO DE GEOGRAFIA E ESTATÍSTIA, 2009).

Acredita-se que o maior número de consultas de pré-natal está fortemente associado a desfechos mais favoráveis (DOMINGUES et al., 2012). Entre as prováveis justificativas que explicariam o número inferior de consultas pré-natais por gestante, encontra-se a escolaridade. É importante salientar que essa variável é um fator diferencial nesse processo de acesso à assistência pré-natal. A educação, nesse caso, revela-se como fator importante na percepção da importância da assistência à saúde maternoinfantil (INSTITUTO BRASILEIRO DE GEOGRAFIA E ESTATÍSTICA, 2009).

A respeito da etnia das mulheres pesquisadas, em sua maioria $(83,1 \%)$, eram negras ou pardas. Esses números estão de acordo com a literatura para gestantes da região Norte e Nordeste do Brasil (INSTITUTO BRASILEIRO DE GEOGRAFIA E ESTATÍSTICA, 2009). De acordo com Cunha, (2008), as gestantes negras apresentam hipertensão arterial de forma mais precoce, mais frequente e mais grave sendo a principal causa de morte materna e responsável por mais de um terço dessas mortes. Por isso a população estudada apresenta perfil de risco para essa doença e assim deve ser triada com mais 
frequência de forma a reduzir as complicações inerentes desse quadro. Quanto ao risco de desenvolver diabetes mellitus - tipo II, gestantes negras têm $50 \%$ de chances a mais do que outras (CUNHA, 2008). Tais informações sugerem que as gestantes pesquisadas no município devem ser acompanhadas com mais cuidado e frequência, e orientadas a respeito de medidas profiláticas para essa doença, devido à sua predisposição natural.

Quanto à faixa etária das gestantes, os dados $(23,6 \%)$ corroboram a literatura que estima a existência no Brasil de cerca de um milhão de adolescentes entre 10 e 20 anos dando a luz todos os anos, o que corresponde a $20 \%$ do total de nascimentos (SPINDOLA et al., 2006; BUENDGENS; ZAMPIERI, 2012; COSTA et al., 2013). No mundo, cerca de 16 milhões de mulheres entre 15 e 19 anos engravidam a cada ano. Na América latina, o percentual gira em torno de $18 \%$ e metade deste ocorre em apenas sete países, um deles o Brasil (INSTITUTO BRASILEIRO DE GEOGRAFIA E ESTATÍSTICA, 2009; WHO, 2014). Embora os partos das adolescentes entre 10 e 19 anos representem $11 \%$ de todos os nascimentos no mundo, $23 \%$ do total das morbidades estão associadas ou decorrem da gravidez de adolescentes, tendo essas complicações mais graves do que as mulheres adultas. $\mathrm{Na}$ América Latina, o risco de morte materna é quatro vezes maior entre as adolescentes menores de 16 anos do que na faixa dos vinte anos (SILVA; SURITA, 2009; WHO, 2014).

A prevalência da gravidez em mulheres com idade acima de 40 anos também mostrou-se semelhante ao restante do país (INSTITUTO BRASILEIRO DE GEOGRAFIA E ESTATÍSTICA, 2009). De acordo com dados da literatura, o risco de morte materna é cinco vezes maior após os 40 anos (SILVA; SURITA, 2009). Isso pode ser devido às complicações gestacionais inerentes ao envelhecimento.

Quanto ao tipo de parto, foi observado que 62,6\% das pesquisadas foram submetidas ao perto cesáreo. Esses dados são superiores aos relatados pelo ministério da saúde ao afirmar que a cesariana já representa $43 \%$ dos partos realizados no Brasil nos setores público e privado e que no Sistema Único de Saúde as cesáreas somam 26\% (VICTORA, 2011). Os fatores envolvidos com essa variável são polêmicos e diversos, porém se destaca o medo das gestantes em se submeterem ao parto normal e conveniência para o médico quanto à adequação aos seus horários de trabalho. A recomendação da Organi- zação Mundial da Saúde é para que as cirurgias cesáreas sejam, no máximo, 15\% do total dos partos, limitando-se a situações de risco tanto da mãe quanto da criança (INSTITUTO BRASILEIRO DE GEOGRAFIA E ESTATÍSTICA, 2009).

Quanto à presença de aborto espontâneo prévio, foi observado que aproximadamente $20 \%$ das mulheres pesquisadas já haviam passado por esse trauma, dado esse superior aos relatados em outras partes do Brasil, onde, segundo o IBGE, $14 \%$ das brasileiras referiram alguma vez ter tido aborto espontâneo prévio (CECATTI et al., 2010; BORSARI et al., 2013; BARBARESCO et al., 2014). A taxa de abortos aumentou com a idade das mulheres, com as maiores proporções sendo registradas entre aquelas de mais de 35 anos de idade (3,9\%) (CECATTI et al., 2010). Com o envelhecimento, vários fatores podem interferir no desenvolvimento adequado do embrião, destacando-se a exposição dos óvulos a fatores ambientais por mais tempo, alteração no endométrio uterino, que pode refletir na nutrição deficiente do feto.

É consenso na literatura que o aborto espontâneo é a mais comum das complicações da gravidez (SILVA; SURITA, 2009; BORSARI et al., 2013; WHO, 2014; BARBARESCO et al., 2014; DE-PASCHALE et al., 2014). Porém, a história reprodutiva de 3 ou mais perdas espontâneas sucessivas de abortamento até 20 semanas de gestação pode ser caracterizado como aborto recorrente e ocorre em 1 a $2 \%$ das mulheres em fase procriativa, dados semelhantes aos encontrados nessa pesquisa (COSTA et al., 2013).

O aborto espontâneo pode ter etiologia multifatorial, como infecções congênitas causadas pelos microrganismos (Toxoplasma gondii, Trypanosoma cruzi, Vírus da rubéola, Citomegalovírus, Treponema pallidum, entre outros agentes infecciosos), anomalias cromossômicas e baixos níveis de progesteronas (BORSARI et al., 2013; BARBARESCO et al., 2014).

No Brasil, doenças infecciosas durante a gravidez são relativamente frequentes, afetando especialmente populações menos favorecidas (PADMAVATHY et al., 2013). Tal situação gera desafios à saúde pública para planejar estratégias de triagem dessas doenças de modo prático e abrangente, facilitando o manejo clínico das gestantes com o diagnóstico adequado (DE-PASCHALE et al., 2014).

A triagem sorológica para toxoplasmose, doença de Chagas, rubéola, citomegalovírus e sífilis ou para 
síndrome STORCH (sífilis, toxoplasmose, rubéola, citomegalovírus e herpes vírus) é de suma importância na gestação, pois possibilita o tratamento precoce, evitando a ocorrência de aborto, malformações congênitas e/ou problemas tardios (AVELINO et al., 2014).

Nesse trabalho, foram encontrados todos os agentes causadores da síndrome STORCH [Sífilis (Treponema pallidum), Toxoplasmose (Toxoplasma gondii), Rubéola, citomegalovírus (CMV), herpes simplex vírus (HSV)] e outros agentes como, Neisseria gonorrhoeae, HIV. Esses agentes infecciosos causam quase sempre infecção assintomática ou leve na mãe, mas pode desencadear graves problemas no feto (BARRETO et al., 1987; PADMAVATHY et al., 2013; MANICKLAL et al., 2013; OHASHI et al., 2014). A prevalência da toxoplasmose entre as infecções congênitas é significativamente maior às outras infecções, dado concordante com o encontrado (BARRETO et al., 1987). Estudos soroepidemiológico mostram que de 10 a 20\% das mulheres brasileiras são suscetíveis à rubéola e nesse trabalho foi encontrado uma gestante agudamente infectada (PADMAVATHY et al., 2013). Essa infecção pode desencadear malformação congênita entre 10-54\% das vezes. A citomegalovirose congênita é doença viral mais comum no período gestacional e tem uma incidência que varia de 0,5 a $3 \%$ dos recém-nascidos, dado superior ao encontrado nesse inquérito (MANICKLAL et al., 2013). Os herpes simples na parte inicial da gravidez são relacionados a aborto espontâneo e à malformação congênita (PADMAVATHY et al., 2013). Quanto às sífilis gestacional, a prevalência encontrada nesse inquérito se assemelha ao outros estudos em outras partes do Brasil (BARRETO et al., 1987), porém apresenta maior prevalência do que o trabalho de (DE-PASCHALE et al., 2014) na África.

Quanto à infecção pelo vírus HIV, a prevalência encontrada de 1,9\% é superior à encontrada em um Estudo Sentinela-Parturiente, conduzido pelo então Programa Nacional de DST e AIDS (atual Departamento de DST, Aids e Hepatites Virais), que estimou a prevalência da infecção pelo HIV nessa população em $0,41 \%$ (BRASIL, 2010b). Assim, o grupo estudado prevalência 4.63 vezes maior que a Brasileira. Foi observado correlação entre a infecção pelo vírus HIV e infecção urinária, assim as mulheres HIV+ apresentaram 9,38 vezes mais infecção urinária que as outras (OR: 9,3811; IC:3,7654-23,3721).

Quanto às hepatites B e C, os valores encontrados foram semelhantes aos relatados em outros estudos
(FERNANDES et al., 2014; DE-PASCHALE et al., 2014, GOIÁS, 2012).

Quanto à infecção urinária, a prevalência encontrada foi semelhante aos de outros trabalhos, que afirmam que estas acometem aproximadamente $10 \%$ internações hospitalares nesse grupo (BAUMGARTEN et al., 2011). Quanto à Hipertensão, a prevalência (15,6\%) encontrada é semelhante às relatadas por outros inquéritos no Brasil (FREITAS et al., 2005; SPINDOLA et al., 2006; CUNHA, 2008; BRASIL, 2010a; BARRA et al., 2012). Diferentemente dos países desenvolvidos, a hipertensão arterial na gestação permanece a primeira causa de morte materna direta no Brasil (37\%), sendo a proporção maior nas regiões Norte e Nordeste em relação ao Sudeste, Sul e Centro-Oeste (FREIRE; TEDOLDI, 2009).

As síndromes hipertensivas da gravidez são a principal causa de morbimortalidade materno-fetal no mundo desenvolvido, ocorrendo em cerca de $8 \%$ das gestantes. (BARRA et al., 2012).

Foi encontrada correlação entre hipertensão e a idade, dado concordante com relatos da literatura brasileira que afirmam que, nas mulheres em idade procriativa, a prevalência vai de 0,6 a 2,0\%, na faixa etária de 18 a 29 anos, e de 4,6 a 22,3\%, na faixa etária de 30 a 39 anos (BARRA et al., 2012).

Sobre o diabetes gestacional, incidência em diferentes inquéritos no Brasil varia de $3 \%$ a $7 \%$, de acordo com a população estudada e com os critérios diagnósticos utilizados (LENZ; FLORES, 2011; SOCIEDADE BRASILEIRA DE ENDOCRINOLOGIA E METABOLOGIA, 2008; SPINDOLA; PENNA; PROGIANTI, 2006; WHO, 2014). Números superiores ao encontrado nesse inquérito (1,1\%). Foi encontrado correlação entre o diabetes gestacional e a infecção urinária. As mulheres diabéticas tinham 356,24\% a mais de casos de infecção Urinária (OR:4,5624; IC:1,1570-17,9915).

Quanto às cardiopatias, os valores encontrados (1\%) são inferiores aos relatados no Brasil. A importância dos estudos sobre as cardiopatias na gravidez justifica-se por dois aspectos: a incidência média de cardiopatia na gravidez é de $4,2 \%$, oito vezes maior quando comparada às médias internacionais e, além disso, a cardiopatia é considerada a maior causa de morte materna indireta no ciclo gravídico-puerperal. Portanto, é importante que sejam estabelecidas diretrizes para o atendimento da mulher cardiopata, tanto na gravidez quanto no planejamento familiar (LAGE; BARBOSA, 2012). 
Em relação às queixas registradas no atendimento obstétrico do pré-natal, os dados mostram que a maioria das gestantes $(82,15 \%)$ relatara queixas na gestação, dados convergentes com os de Spindola; Penna e Progianti, (2006) em pesquisa feita em um Hospital universitário do Rio de Janeiro onde a maioria das gestantes $(66,75 \%$ 79/118). Nesse trabalho as principais queixas foram: cefaleia $(38,0 \%)$, Dor no baixo-ventre $(35,5 \%)$ e leucorreia $(16,9 \%)$ e na pesquisa de Spindola; Penna e Progianti, (2006) 22,88\% relataram dor em baixo-ventre sem perdas transvaginais; $19,49 \%$ náuseas e 12,71\% cefaleia não associada à Hipertensão Arterial (SPINDOLA et al., 2006). Esses dados evidenciam o perfil das pacientes e confirmam a importância da assistência pré-natal que compreende todas as medidas introduzidas e/ou recomendadas durante a gestação com objetivo de preservar a saúde materno-fetal e reduzir a morbi-mortalidade do binômio mãe-filho.

\section{Conclusões}

O perfil das gestantes atendidas é de mulheres jovens, com baixa escolaridade, casadas, que não exercem atividade remunerada, multíparas, com renda familiar de um salário mínimo, submetidas a parto cesáreo. Essas informações mostram a importância de campanhas educativas de orientação para mulheres dessa faixa etária e renda. Mostra-se fundamental e urgente que sejam tomadas medidas de convencimento das gestantes e equipe médica, quanto as vantagens do parto normal.

Quanto às infecções gestacionais, estão presentes a toxoplasmose, AIDS, citomegalovírus, Hepatites B e C, Rubéola, sífilis. Quanto às doenças não infecciosas, destacam-se a hipertensão, diabetes, cardiopatia, encefalopatia e doenças renais. A maioria referiu queixas na gestação e não apresentava patologias associadas ao processo gestacional.

Acredita-se que devido à fragilidade da saúde materna na gestação se torna importante a intensificação ou a participação da equipe de saúde no acompanhamento das gestantes de baixo risco de forma que sejam tomadas medidas profiláticas e curativas impedindo ou minorando o surgimento de gestações de alto risco. A prevenção de complicações obstétricas e as ações educativas desenvolvidas no pré-natal são essenciais para o acompanhamento e
Este trabalho de caracterizar a população de gestantes de Gurupi se mostra oportuno em princípio, e seus resultados servirão de base para a ampliação gradativa, dos serviços de atenção básica e especializada a nível regional.

\section{Referências}

ANDREUCCI, C.; CECATI, J. Desempenho de indicadores de processo do Programa de Humanização do Pré-natal e Nascimento no Brasil: uma revisão sistemática. Cadernos de Saúde Pública, Rio de Janeiro, v. 27, n. 6, p. 1053-1064, jan./jun. 2011. doi:10.1590/S0102311X2011000600003.

AVELINO, M. M. et al. Congenital toxoplasmosis and prenatal care state programs. BMC Infectious Diseases, London, v. 14, n. 33, p. 1-13, jan. 2014. doi:10.1186/14712334-14-33.

BARBARESCO, A. A. et al. Vertical transmission from abortive material and blood with emphasis on Toxoplasma gondii. Revista Brasileira de Ginecologia e Obstetrícia, Rio de Janeiro, v. 36, n. 1, p. 17-22, jan. 2014. doi: 10.1590/S0100-72032014000100005.

BARRA, S. et al. Hypertension in pregnancy: the current state of the art. Revista Portuguesa de Cardiologia, Lisboa, v. 31, n. 6, p. 425-432, jun. 2012.

BARRETO, S. M. V.; COSTA, J. C.; GONCALVES, A. L. Pesquisa de anticorpos para sífilis e toxoplasmose em recém-nascidos em hospital de Ribeirão Preto, SP, Brasil. Revista de Saúde Pública, São Paulo, v. 21, n. 1, p. 55-63, 1987. doi: 10.1590/S0034-89101987000100009.

BARROS, S. M. O. Enfermagem no ciclo gravídico-puerperal. São Paulo: Manole, 2006.

BAUMGARTEN, M. C. S. et al. Infecção urinária na gestação: uma revisão da literatura. Unopar Científica Ciências Biológicas e da Saúde, Londrina, v. 13, n. 1, p. 333-342, jan./mar. 2011.

BORSARI, C. M. G. et al. Abortion in women living in the outskirts of São Paulo: experience and socioeconomic aspects. Revista Brasileira de Ginecologia e Obstetrícia, Rio de Janeiro, v. 35, n. 1, p. 27-32, jan. 2013. doi: 10.1590/S0100-72032013000100006. 
BRASIL. Ministério da Saúde. Aborto e saúde pública no Brasil: 20 anos. Brasília: MS, 2009.

BRASIL. Ministério da Saúde. Atenção ao pré-natal de baixo risco. 2012. Disponível em: <http://bvsms.saude. gov.br/bvs/publicacoes/cadernos_atencao_basica_32_ prenatal.pdf>. Acesso em: 22 set. 2015.

BRASIL. Ministério da Saúde. Gestação de alto risco: manual técnico. Brasília: MS, 2010a.

BRASIL. Ministério da Saúde. Recomendações para profilaxia da transmisão vertical do HIV e terapia antirretroviral em gestantes: manual de bolso. Brasília: Ministério da Saúde, 2010b.

BUENDGENS, B. B.; ZAMPIERI, M. F. M. A adolescente grávida na percepção de médicos e enfermeiros da atenção básica. Escola Anna Nery, Rio de Janeiro, v. 16, n. 1, p. 6472, mar. 2012. doi: 10.1590/S1414-81452012000100009.

CECATTI, J. G. et al. Aborto no Brasil: um enfoque demográfico. Revista Brasileira de Ginecologia e Obstetricia, Rio de Janeiro, v. 32, n. 3, p. 105-111, mar. 2010. doi: 10.1590/S0100-72032010000300002.

\section{CONSELHO NACIONAL DE SAÚDE. Diretrizes e nor-} mas regulamentadoras de pesquisas envolvendo seres humanos: Resolução nº196/96. Brasília: MS, 2006.

COSTA, C. S. C. et al. O. Características do atendimento pré-natal na Rede Básica de Saúde. Revista Eletrônica de Enfermagem, Goiânia, v. 15, n. 2, p. 516-522, abr./jun. 2013. doi: $10.5216 /$ ree.v15i2.15635.

COSTA, E. S. et al. Alterações fisiológicas na percepção de mulheres durente a gestação. Revista da Rede de Enfermagem do Nordeste, Fortaleza, v. 11, n. 2, p. 86-93, abr./jun. 2010.

COUTINHO, T. et al. Monitoramento do processo de assistência pré-natal entre as usuárias do Sistema Único de Saúde em município do Sudeste brasileiro. Revista Brasileira de Ginecologia e Obstetricia, Rio de Janeiro, v. 32 , n. 11 , p. 563-569, nov. 2010. doi: 10.1590/S010072032010001100008 .

CUNHA, E. M. G. D. P. O recorte racial no estudo das desigualdades em saúde. São Paulo em Perspectiva, São Paulo, v. 22, n. 1, p. 79-91, jan./jun. 2008.
DE-PASCHALE, M. et al. Antenatal screening for Toxoplasma gondii, Cytomegalovirus, rubella and Treponema pallidum infections in northern Benin. Tropical Medicine \& International Health, Oxford, v. 19, n. 6, p. 743746, jun. 2014. doi: 10.1111/tmi.12296.

DOMINGUES, R. M. S. M. et al. Avaliação da adequação da assistência pré-natal na rede SUS do Município do Rio de Janeiro , Brasil. Cadernos de Saúde Pública, Rio de Janeiro, v. 28, n. 3, p. 425-437, mar. 2012. doi: 10.1590/ S0102-311X2012000300003.

FERNANDES, C. N. D. S. et al. Prevalência de soropositividade para hepatite B e C em gestantes. Revista da Escola de Enfermagem da USP, São Paulo, v. 48, n. 1, p. 89-96, jan./fev. 2014. doi: 10.1590/S0080-623420140000100011.

FREIRE, C. M. V.; TEDOLDI, C. L. Hipertensão arterial na gestação. Arquivos Brasileiros de Cardiologia, São Paulo, v. 93, n. 6, p. 159-165, dec. 2009. doi: 10.1590/ S0066-782X2009001300017.

FREITAS, P. F. et al. Desigualdade social nas taxas de cesariana em primíparas no Rio Grande do Sul. Revista de Saúde Pública, São Paulo, v. 39, n. 5, p. 761-767, oct. 2005. doi: 10.1590/S0034-89102005000500010.

GOIÁS. Secretaria de Saúde. Plano Estadual de Saúde 2012-2015. Goiânia; 2012. Disponível em: <http://www. sgc.goias.gov.br/upload/links/arq_944_planoestadualdesaude2012-2015.pdf>. Acesso em: 22 set. 2015.

INSTITUTO BRASILEIRO DE GEOGRAFIA E ESTATÍSTICA. Indicadores sociodemográficos e de saúde no Brasil. Rio de Janeiro: IBGE, 2009a.

INSTITUTO BRASILEIRO DE GEOGRAFIA E ESTATÍSTICA. Síntese de indicadores sociais: uma análise das condições de vida da população brasileira. Rio de Janeiro: IBGE, 2013.

INSTITUTO DE PESQUISA ECONÔMICA APLICADA. Atlas de desenvolvimento humano no Brasil, 2013: perfil do município de Gurupi, TO. 2013. Disponível em: $<$ http://atlasbrasil.org.br/2013/perfil_print/gurupi_to $>$. Acesso em: 22 set. 2013. 
LAGE, E. M.; BARBOSA, A. S. Cardiopatias e gravidez. FEMINA, Goiânia, v. 40, n. 1, p. 42-50, jan./fev. 2012.

LEE, M. et al. Urinary tract infections in pregnancy. Canadian Family Physician, Ontario, v. 54, n. 6, p. 853-854, jun. 2008.

LENZ, M. L. M.; FLORES, R. Atenção à saúde da gestante em APS. Porto Alegre: Hospital Nossa Senhora da Conceição, 2011.

LUPPI, P. et al. Monocytes are progressively activated in the circulation of pregnant women. Journal of Leukocyte Biology, Bethesda, v. 72, n. 5, p. 874-884, nov. 2002.

MANICKLAL, S. et al. The "silent" global burden of congenital cytomegalovirus. Clinical Microbiology Reviews, Washington, v. 26, n. 1, p. 86-102, jan. 2013. doi: 10.1128/CMR.00062-12.

NEVES, C.; MEDINA, J. L.; DELGADO, J. L. Alterações endócrinas e imuno-modulação na gravidez. Arquivos de Medicina, Porto, v. 21, n. 5/6, p. 175-182, out./dec. 2007.

OHASHI, K. et al. Viral load before and after exchange transfusion in a neonate with hyperbilirubinemia and congenital cytomegalovirus infection. Journal of Clinical Case Reports, Miami, v. 4, n. 3, 43, p. 1-2, mar. 2014. doi: 10.4172/2165-7920.1000343.

PADMAVATHY, M. et al. Seroprevalence of TORCH infections and adverse reproductive outcome in current pregnancy with bad obstetric history. Journal of Clinical and Biomedical Sciences, India, v. 3, n. 2, p. 62-71, abr./ jun. 2013.

PORTUGAL. Direcção-Geral da Saúde Divisão de Saúde Materna, Infantil e dos Adolescentes. Saúde Reprodutiva: doenças Infecciosas e gravidez / Direcção-Geral da Saúde. Lisboa: Direcção-Geral da Saúde, 2000. Disponível em: <http://www.dgs.pt/upload/membro.id/ficheiros/ ioo8092.pdf>. Acesso em: 01 out. 2015

PROENÇA, C. O sistema imunológico na gestação. Revista de Pesquisa Médica, 2011. Disponível em: <http:// www.revistapesquisamedica.com.br/PORTAL/textos.asp? codigo $=11631>$. Acesso em: 22 set. 2015.
SILVA, J. L. C. P.; SURITA, F. G. C. Idade materna: resultados perinatais e via de parto. Revista Brasileira de Ginecologia e Obstetricia, Rio de Janeiro, v. 31, n. 7, p. 321325, jul. 2009. doi: 10.1590/S0100-72032009000700001.

SOCIEDADE BRASILEIRA DE ENDOCRINOLOGIA E METABOLOGIA. Diabetes mellitus gestacional. Revista da Associação Médica Brasileira, São Paulo, v. 54, n. 6, p. 477-480, nov./dec. 2008. doi: 10.1590/S010442302008000600006.

SPINDOLA, T.; PENNA, L. H. G.; PROGIANTI, J. M. Perfil epidemiológico de mulheres atendidas na consulta do pré-natal de um hospital universitário. Revista da Escola de Enfermagem da USP, São Paulo, v. 40, n. 3, p. 381-388, jul./set. 2006.

VIANNA, P. Imunorregulação da gestação: rumo ao sucesso. 2009. 165 f. Tese (Doutorado) - Programa de Pós Graduação em Genética e Biologia Molecular da Universidade Federal do Rio Grande do Sul, Porto Alegre, 2009.

VICTORA, C.G. et al. Maternal and child health in Brazil: progress and challenges. Lancet, London, v. 377 , n. 9780, p. 1863-1876, may 2011. doi: 10.1016/S01406736(11)60138-4.

WORLD HEALTH ORGANIZATION (WHO). Managing complications in pregnancy and childbirth (MCPC): a guide for midwives and doctors. Geneva: WHO, 2010. Disponível em: <http://www.who.int/en/>. Acesso em: 01 out. 2015.

WORLD HEALTH ORGANIZATION (WHO). Adolescent pregnancy. Fact sheet $N^{\circ} 364$ Updated September 2014. Geneva: WHO. Disponível em: <http://www.who.int/mediacentre/factsheets/fs364/ en/>. Acesso em: 01 out. 2015.

ZIEGEL, E. E.; CRANLEY, M. S. Enfermagem obstétrica. Tradução J. Israel Lemos T. E. G. O. 8. ed. Rio de Janeiro: Guanabara Koogan, 1985. 